\title{
Effect of the two antioxidants; Glutathione and ascorbic acid on vegetative growth, yield and some biochemical changes in two wheat cultivars
}

\author{
Mohamed El-Sayed El-Awadi, Safaa Reda El-Lethy, Kawther Gad El-Rokiek \\ Botany Department, National Research Centre, Dokki, Giza, Egypt \\ Email address: \\ el_awadi@yahoo.com (M. E. El-Awadi), safaa.ellethy@yahoo.com (S. R. El-Lethy), ahmed_ezat2000@yahoo.com (K. G. El-Rokiek)
}

\section{To cite this article:}

Mohamed E. El-Awadi, Safaa R. El-Lethy, Kawther G. El-Rokiek. Effect of the Two Antioxidants; Glutathione and Ascorbic Acid on Vegetative Growth, Yield and Some Biochemical Changes in Two Wheat Cultivars. Journal of Plant Sciences. Vol. 2, No. 5, 2014, pp. $215-221$. doi: $10.11648 /$ j.jps.20140205.20

\begin{abstract}
Two field experiments were carried out in the experimental farm of National Research Center at Nubaria, Egypt, during two successive seasons 2011/2012 and 2012/2013 to study the effect of the two antioxidants; glutathione and ascorbic acid on growth, yield and yield components of two cultivars of wheat plant (Triticum aestivum L.). The two cultivars were Sakha93 and Giza168. The two antioxidants were foliarly applied twice at the two concentrations 50 and $100 \mathrm{ppm}$ for each. The first spray was applied 30 days after sowing and the second spray was applied 15 days later (45 days after sowing). The results indicated significant increases in the growth of the two wheat cultivars Sakha93 and Giza168. The increase in growth was accompanied by increase in yield and yield components of the two cultivars compared to the corresponding controls. In general, growth and yield exhibited greatest response towards the higher concentration of glutathione. The increase in wheat growth was concomitant with increases in photosynthetic pigments, carbohydrate, total free amino acids and protein contents. The results suggested using both antioxidants glutathione and ascorbic acid especially at $100 \mathrm{ppm}$ of glutathione to improve wheat growth and yield.
\end{abstract}

Keywords: Triticum Aestivum L., Wheat Cultivars, Glutathione, Ascorbic Acid

\section{Introduction}

An antioxidant is a molecule that inhibits the oxidation of other molecules. Oxidation reactions can produce free radicals. In turn, these radicals can start chain reactions in cell and can cause damage or death to the cell. Antioxidants terminate these chain reactions by removing free radical intermediates, and inhibit other oxidation reactions [1 and 2]. Ascorbic acid and glutathione are of the most important antioxidants.

Ascorbic acid is a naturally occurring organic compound with antioxidant properties. Ascorbic acid was synthesized in higher plants and considered an important antioxidant; it is the most abundant antioxidant currently considered to be a regulator on cell division and differentiation, photoprotection and regulation of photosynthesis and growth, played an important role as co-enzymatic reaction in the electron transport system and metabolism [1 and 3].

Study on the effect of ascorbic acid on wheat in new reclaimed soil showed increase in plant height and spike length and caused increase in grain yield when mixed with cupper [4]. Amin et al. [5] and Bakry et al. [6] reported that treating wheat with ascorbic acid up to $400 \mathrm{ppm}$ increased plant height, number of tillers and spikes, flag leaf area, grain index, grain and straw yield per plant and per feddan as well as some biochemical constituents.

Ascorbic acid antagonized hardening of wheat grains at 45 and $55^{\circ} \mathrm{C}$ where it caused increase in vegetative growth and yield [7]. Similar effect of ascorbic acid was found by Amin et al. [8] on drought stress of okra (Hibiscus esculentus L.).

Glutathione $(\mathrm{GSH})$ is a tripeptide with a gamma peptide linkage between the group of cysteine (which is attached by normal peptide linkage to a glycine) and the carboxyl group of the glutamate side-chain. It is an antioxidant, preventing damage to important cellular components caused by reactive oxygen species such as free radicals and peroxides [9]. In plants, glutathione acts in the ascorbate-glutathione cycle to maintain the cellular redox homeostasis in response to oxidative stresses. In this cycle, GSH is used by dehydroascorbate reductase (DHAR) to regenerate ascorbate to scavenge $\mathrm{H}_{2} \mathrm{O}_{2}$, and is converted to oxidized form (GSSG), 
which is then regenerated by glutathione reductase (GR) [10 and 11]. Hence, the capacity of glutathione to participate in the redox regulation depends to a great extent on its absolute concentration and the ratio of GSH/GSSG [12, 13, 14]. Glutathione is also a regulator of plant development. Flowering of Arabidopsis thaliana was affected by glutathione synthesis and levels [14].

In the present study was carried out to compare the effect of ascorbic acid and glutathione on vegetative growth, yield components and changes in some biochemical compounds of two wheat cultivars (Sakha 93 and Giza 168) cultivated at new reclaimed area (Nubaria, North western of Egypt).

\section{Materials and Methods}

This investigation was carried out at the experimental farm of National Research Center at Nubaria, Egypt, during two successive seasons 2011/2012 and 2012/2013 to study the effect of glutathione and ascorbic acid on growth, development, yield and yield components and main biochemical components of two cultivars of wheat plant (Triticum aestivum L.).

\subsection{Plant Materials}

Uniform grains of two local cultivars of wheat plant (Triticum aestivum L.) Sakha93 and Giza168 were obtained from Wheat Research Department, Agricultural Research Center, Ministry of Agriculture, Egypt.

The experiments were carried out under sandy soil conditions (Table 1). Grains of the two cultivars were sown at 27 and 20 November for the first and the second season respectively. The plot area was $10.5 \mathrm{~m}^{2}$ (1/400 of feddan), three meters in length and three and half meters in width. Seeds were sown at the rate of $60 \mathrm{Kg} /$ feddan. The experimental design was split plot design with three replicates. The two cultivars were assigned in the main plot and the treatments of the antioxidants were distributed at random in the subplot.

Nitrogen fertilizer was applied at the rate of 120units $\mathrm{N} /$ feddan in the form of ammonium nitrate $(33.5 \% \mathrm{~N})$, it was divided in three equal portions (started at 20day after sowing). The dosage was added before the irrigation. Phosphorus fertilizer was applied before sowing (during land preparation) at rate of $150 \mathrm{Kg} \mathrm{P}_{2} \mathrm{O}_{5}$ /feddan in the form of calcium super phosphate $\left(15.5 \% \mathrm{P}_{2} \mathrm{O}_{5}\right)$. Potassium sulphate was added at rate of $50 \mathrm{Kg}$ per feddan, and applied one month after sowing.

Glutathione and ascorbic acid were foliarly applied at two concentrations (50 and100 ppm). First spray was carried out at vegetative stage (30 days after sowing) the spray volume of this sprinkling was 200 liter per feddan, and the second spray was fifteen days later its volume was 200 liters per feddan. Plant Samples were drawn at both vegetative stage (60 days after sowing) and at heading stage (90 days after sowing).

Growth criteria were measured; plant height $(\mathrm{cm})$, number of leaves per plant, fresh weight of shoot per plant and dry weights of shoot per plant were determined after drying in electric oven at $70^{\circ} \mathrm{C}$.

Table 1. Physical and chemical analysis of the soil at Nubaria region.

\begin{tabular}{|c|c|c|c|c|c|c|c|c|c|c|c|c|}
\hline \multicolumn{3}{|c|}{ Soil particles \% } & \multirow{2}{*}{$\begin{array}{l}\text { Texture } \\
\text { type }\end{array}$} & \multirow{2}{*}{$\begin{array}{l}\text { Ec. Conductivity at } \\
25^{\circ} \mathrm{C}\left(\mathrm{mmhos} / \mathrm{cm}^{2}\right)\end{array}$} & \multirow{2}{*}{ pH } & \multirow{2}{*}{$\begin{array}{l}\text { Organic } \\
\text { matter (\%) }\end{array}$} & \multirow{2}{*}{$\begin{array}{l}\text { Ca } \\
\text { Meq/l. }\end{array}$} & \multirow{2}{*}{$\begin{array}{l}\text { Mg } \\
\text { Meq/l. }\end{array}$} & \multirow{2}{*}{$\begin{array}{l}\text { Na } \\
\text { Meq/l. }\end{array}$} & \multirow{2}{*}{$\begin{array}{l}\text { K } \\
\text { Meq/l. }\end{array}$} & \multirow{2}{*}{$\begin{array}{l}\mathrm{HCO}_{3} \\
\mathrm{Meq} / \mathrm{l} .\end{array}$} & \multirow{2}{*}{$\begin{array}{l}\text { Cl } \\
\text { Meq/l. }\end{array}$} \\
\hline Sand & Silt & Clay & & & & & & & & & & \\
\hline 90.08 & 0.66 & 9.26 & Sandy & 1.7 & 8.2 & 0.24 & 7.02 & 0.53 & 0.98 & 0.31 & 1.30 & 0.57 \\
\hline
\end{tabular}

\subsection{Yield Measurements}

At harvest, ten plants from each replicate were randomly chosen to determine the following criteria:
a Number of spikes /plant
b Spike length $(\mathrm{cm})$.
c Number of spiklets per spike
d Number of grains per spike.
e Grain weight per spike (g).
f Grain weight /plant (g).
$\mathrm{g}$ Weight of 1000grains
$\mathrm{h}$ Grain yield per feddan $(\mathrm{Kg})$.

\subsection{Biochemical Compounds Determination}

Photosynthetic pigments were measured in fresh tissues of wheat leaves according to Wettstein [15] and A.O.A.C. [16]. Protein percentage was determined according to A.O.A.C. [17]. Total Free amino acids were determined using the ninhydrin colorimetric method defined by Plummer [18]. Following the method reported by Snell and Snell [19], total phenolic compounds were determined. Total carbohydrates were determined according to Dubois et al. [20].

\subsection{Statistical Analysis}

The experimental design was split block with three replicates. The data obtained were submitted to standard analysis of variance procedure of design; LSD values were obtained when $\mathrm{F}$ values were significant at 5\% level [21].

\section{Results}

The data in Table 2 show significant increases in plant height in the two wheat cultivars due to the two antioxidants glutathione (Glut.) and ascorbic acid (ASA). Different significant responses were detected between the two cultivars according to the treatments with the two antioxidants. Different effects also were identified according to antioxidant type and concentration. Plant height at vegetative stage increased with increase in Glut. concentration, while ASA induced higher increase at lower concentration (50ppm). The increase was consistent at heading stage. Number of leaves as 
well exhibited relatively less response at both vegetative and heading stages. The treatments of Glut. or ASA at the two concentrations $(50 \& 100 \mathrm{ppm})$ caused significant increases in fresh weight of shoots of both wheat cultivars Sakha 93 and Giza 168 at both vegetative and heading stages (Table 3 ) as compared to corresponding controls. The increase in fresh weight was concomitant by increase in dry matter. Maximum significant increase in dry weight of shoot was recorded with foliar application of Glut. at $100 \mathrm{ppm}$.

\subsection{Yield and its Components}

Significant differences between the two wheat cultivars in yield and its components due to Glut. or ASA treatments (Tables4\&5). The results indicate significant increase in number of spikes/plant, spike length and number of spiklets/spike with Glut. or ASA in the two wheat cultivars. These increases were higher at treatment100 ppm Glut. in comparison to the corresponding controls (Table 4).

The treatments of the two wheat cultivars with Glut. or ASA resulted in significant increases in weight of grains/spike, weight of grains/plant, weight of 1000 grains (g) as well as grain yield $(\mathrm{kg} / \mathrm{fed}$.$) in comparison to the corresponding$ controls (Table 5). The treatment of Glut. at 100ppm exceeded the other treatments. The trend of increase was similar in the two cultivars due to the antioxidants, Glut. recorded $50 \%$ increase over the control in yield( $\mathrm{Kg} /$ feddan) in the cultivar Sakha 93. The corresponding results in the cultivar Giza 168 was $40.9 \%$ over the control (Table 5).

\subsection{Biochemical Changes}

The data in Table 6 reveal significant differences between the two cultivars in chlorophylls a \& b and carotenoids as indicated by the mean values. Increases in the synthesis of chlorophyll a, chlorophyll $\mathrm{b}$ and carotenoids in the leaves of the two wheat cultivars with foliar treatments of Glut. or ASA were obtained in comparison to the corresponding controls.. Increasing concentration rate in Glut. caused higher significant increase, while ASA induced more significant increase with the lower concentration (50ppm). Maximum significant increases in the photosynthetic pigments were obtained with the higher concentration of Glut. (100ppm).

Total free amino acids content exhibited significant increase in the leaves of the two wheat cultivars due to the exogenous application of the two antioxidants Glut. or ASA (Table 7) in comparison to the corresponding controls. Maximum increase was obtained at 100 ppm Glut. in the two cultivars. However, total phenol content significantly increased only at $50 \mathrm{ppm}$ ASA.

Table 2. Effect of the two antioxidants glutathione and ascorbic acid on plant height and number leaves / plant of the two wheat cultivars (Sakha 93 and Giza 168) at both vegetative and heading. (Average of the two seasons).

\begin{tabular}{|c|c|c|c|c|c|c|c|c|c|c|c|c|}
\hline \multirow{3}{*}{ Treatments (ppm) } & \multicolumn{6}{|c|}{ Vegetative stage } & \multicolumn{6}{|c|}{ Heading stage } \\
\hline & \multicolumn{3}{|c|}{ Plant height (cm) } & \multicolumn{3}{|c|}{ No. of leaves } & \multicolumn{3}{|c|}{ Plant height $(\mathrm{cm})$} & \multicolumn{3}{|c|}{ No. of leaves } \\
\hline & $\begin{array}{l}\text { Sakha } \\
93 \\
\end{array}$ & $\begin{array}{l}\text { Giza } \\
168 \\
\end{array}$ & Mean & $\begin{array}{l}\text { Sakha } \\
93 \\
\end{array}$ & $\begin{array}{l}\text { Giza } \\
168 \\
\end{array}$ & Mean & $\begin{array}{l}\text { Sakha } \\
93\end{array}$ & $\begin{array}{l}\text { Giza } \\
168 \\
\end{array}$ & Mean & $\begin{array}{l}\text { Sakha } \\
93 \\
\end{array}$ & $\begin{array}{l}\text { Giza } \\
168 \\
\end{array}$ & Mean \\
\hline Control & 41.4 & 48.1 & 44.75 & 4.5 & 5.0 & 4.8 & 44.2 & 56.6 & 50.4 & 8.8 & 11.1 & 9.95 \\
\hline Glutathione 50 & 51.8 & 53.5 & 52.65 & 4.5 & 5.5 & 5.0 & 56.2 & 62.0 & 59.1 & 13.0 & 11.3 & 12.15 \\
\hline Glutathione 100 & 58.7 & 61.0 & 59.85 & 4.4 & 5.4 & 4.9 & 57.4 & 63.2 & 60.3 & 13.8 & 12.7 & 13.25 \\
\hline Ascorbic acid 50 & 54.5 & 59.1 & 56.80 & 4.9 & 5.9 & 5.4 & 60.8 & 68.8 & 64.8 & 11.5 & 12.4 & 11.95 \\
\hline Ascorbic acid 100 & 50.5 & 57.1 & 53.85 & 4.1 & 5.7 & 4.9 & 56.7 & 60.5 & 58.6 & 11.1 & 11.9 & 11.5 \\
\hline \multirow[t]{2}{*}{ Mean } & 51.38 & 55.76 & & 4.48 & 5.5 & & 55.06 & 62.22 & & 11.64 & 11.88 & \\
\hline & \multicolumn{3}{|c|}{ Cultivars $=0.43$} & \multicolumn{3}{|c|}{ Cultivars $=0.08$} & \multicolumn{3}{|c|}{ Cultivars $=1.24$} & \multicolumn{3}{|c|}{ Cultivars $=\mathrm{NS}$} \\
\hline \multirow[t]{2}{*}{ LSD at $5 \%$} & \multicolumn{3}{|c|}{ Treatments $=2.40$} & \multicolumn{3}{|c|}{ Treatments $=0.31$} & \multicolumn{3}{|c|}{ Treatments $=2.92$} & \multirow{2}{*}{\multicolumn{3}{|c|}{ Treatments $=1.62$}} \\
\hline & \multicolumn{3}{|c|}{ Cultivar $X$ treatments $=3.40$} & \multicolumn{3}{|c|}{ Cultivar X treatments $=0.43$} & \multicolumn{3}{|c|}{ Cultivar $X$ treatments $=4.13$} & & \multicolumn{2}{|c|}{ Cultivar $X$ treatments $=2.30$} \\
\hline
\end{tabular}

Table 3. Effect of the two antioxidants glutathione and ascorbic acid on shoot fresh and dry weight of the two wheat leaves cultivars (Sakha 93 and Giza 168) at both vegetative and heading. (Average of the two seasons).

\begin{tabular}{|c|c|c|c|c|c|c|c|c|c|c|c|c|}
\hline \multirow{3}{*}{ Treatments (ppm) } & \multicolumn{6}{|c|}{ Vegetative stage } & \multicolumn{6}{|c|}{ Heading stage } \\
\hline & \multicolumn{3}{|c|}{ Fresh weight (g) } & \multicolumn{3}{|c|}{ Dry weight (g) } & \multicolumn{3}{|c|}{ Fresh weight (g) } & \multicolumn{3}{|c|}{ Dry weight (g) } \\
\hline & $\begin{array}{l}\text { Sakha } \\
93 \\
\end{array}$ & $\begin{array}{l}\text { Giza } \\
168 \\
\end{array}$ & Mean & $\begin{array}{l}\text { Sakha } \\
93 \\
\end{array}$ & $\begin{array}{l}\text { Giza } \\
168 \\
\end{array}$ & Mean & $\begin{array}{l}\text { Sakha } \\
93 \\
\end{array}$ & $\begin{array}{l}\text { Giza } \\
168 \\
\end{array}$ & Mean & $\begin{array}{l}\text { Sakha } \\
93 \\
\end{array}$ & $\begin{array}{l}\text { Giza } \\
168 \\
\end{array}$ & Mean \\
\hline Control & 10.7 & 13.7 & 12.20 & 4.5 & 4.6 & 4.55 & 21.5 & 28.7 & 25.10 & 6.8 & 10.3 & 8.55 \\
\hline Glutathione 50 & 19.4 & 16.8 & 18.10 & 6.2 & 6.0 & 6.10 & 36.4 & 33.7 & 35.05 & 13.4 & 12.1 & 12.75 \\
\hline Glutathione 100 & 21.6 & 23.3 & 22.45 & 7.1 & 6.8 & 6.95 & 49.0 & 52.7 & 50.85 & 16.0 & 15.4 & 15.7 \\
\hline Ascorbic acid 50 & 19.4 & 20.2 & 19.80 & 6.6 & 8.1 & 7.35 & 40.8 & 42.4 & 41.60 & 14.2 & 14.7 & 14.45 \\
\hline Ascorbic acid 100 & 15.4 & 16.6 & 16.00 & 6.3 & 6.0 & 6.15 & 41.3 & 37.1 & 39.20 & 13.8 & 13.6 & 13.75 \\
\hline Mean & 17.3 & 18.12 & & 6.14 & 6.30 & & 37.80 & 38.92 & & 12.84 & 13.22 & \\
\hline LSD at $5 \%$ & $\begin{array}{l}\text { Cultiva } \\
\text { Treatm } \\
\text { Cultiva }\end{array}$ & $\begin{array}{l}=\mathrm{NS} \\
\mathrm{ts}=2.60 \\
\mathrm{X} \text { treatn }\end{array}$ & $\mathrm{t}=3.68$ & $\begin{array}{l}\text { Cultivar } \\
\text { Treatme } \\
\text { Cultivar }\end{array}$ & $\begin{array}{l}=\mathrm{NS} \\
\mathrm{ts}=0.59 \\
\mathrm{X} \text { treatm }\end{array}$ & $\mathrm{t}=0.84$ & $\begin{array}{l}\text { Cultivar } \\
\text { Treatme } \\
\text { Cultivar }\end{array}$ & $\begin{array}{l}=1.1 \\
\text { ts }=1.8 \\
X \text { treatn }\end{array}$ & $\mathrm{t}=2.6$ & \multicolumn{3}{|c|}{$\begin{array}{l}\text { Treatments }=0.70 \\
\text { Cultivar } \mathrm{X} \text { treatment }=1.1\end{array}$} \\
\hline
\end{tabular}


Table 4. Effect of the two antioxidants glutathione and ascorbic acid on spike components of the two wheat cultivars Sakha 93 and Giza 168. (Average of the two seasons).

\begin{tabular}{|c|c|c|c|c|c|c|c|c|c|c|c|c|}
\hline \multirow[b]{2}{*}{ Treatments (ppm) } & \multicolumn{3}{|c|}{ Spike length (cm) } & \multicolumn{3}{|c|}{ No. Spikes/plant } & \multicolumn{3}{|c|}{ No. spiklets/spike } & \multicolumn{3}{|c|}{ No. grains/spike } \\
\hline & $\begin{array}{l}\text { Sakha } \\
93\end{array}$ & $\begin{array}{l}\text { Giza } \\
168\end{array}$ & Mean & $\begin{array}{l}\text { Sakha } \\
93\end{array}$ & $\begin{array}{l}\text { Giza } \\
168\end{array}$ & Mean & $\begin{array}{l}\text { Sakha } \\
93\end{array}$ & $\begin{array}{l}\text { Giza } \\
168\end{array}$ & Mean & $\begin{array}{l}\text { Sakha } \\
93\end{array}$ & $\begin{array}{l}\text { Giza } \\
168\end{array}$ & Mean \\
\hline Control & 8.01 & 10.30 & 9.15 & 4.33 & 4.14 & 4.23 & 16.87 & 20.17 & 18.52 & 48.49 & 53.00 & 50.74 \\
\hline Glutathione 50 & 8.65 & 10.09 & 9.37 & 6.33 & 6.07 & 6.20 & 16.33 & 19.16 & 17.74 & 49.00 & 52.33 & 50.66 \\
\hline Glutathione 100 & 8.78 & 10.90 & 9.84 & 7.00 & 6.87 & 6.93 & 17.44 & 19.28 & 18.36 & 51.33 & 62.33 & 56.83 \\
\hline Ascorbic acid 50 & 8.57 & 10.83 & 9.70 & 6.10 & 6.02 & 6.06 & 19.05 & 21.00 & 20.02 & 51.00 & 66.00 & 58.50 \\
\hline Ascorbic acid 100 & 8.25 & 10.13 & 9.19 & 5.56 & 5.84 & 5.75 & 17.77 & 19.44 & 18.60 & 45.66 & 59.33 & 52.49 \\
\hline Mean & \multicolumn{3}{|c|}{ Cultivars $=0.19$} & \multicolumn{3}{|c|}{ Cultivars $=0.08$} & \multicolumn{3}{|c|}{ Cultivars $=0.54$} & \multicolumn{3}{|c|}{ Cultivars $=1.51$} \\
\hline \multirow[t]{2}{*}{ LSD at $5 \%$} & \multicolumn{3}{|c|}{ Treatments $=0.23$} & \multicolumn{3}{|c|}{ Treatments $=0.18$} & \multicolumn{3}{|c|}{ Treatments $=0.72$} & \multicolumn{3}{|c|}{ Treatments $=2.09$} \\
\hline & \multicolumn{3}{|c|}{ Cultivar X treatment $=0.33$} & \multicolumn{3}{|c|}{ Cultivar X treatment $=0.24$} & \multicolumn{3}{|c|}{ Cultivar X treatment $=1.01$} & \multicolumn{3}{|c|}{ Cultivar X treatment $=2.96$} \\
\hline
\end{tabular}

Table 5. Effect of the two antioxidants glutathione and ascorbic acid on yield and yield components of the two wheat cultivars Sakha 93 and Giza 168 . (Average of the two seasons).

\begin{tabular}{|c|c|c|c|c|c|c|c|c|c|c|c|c|}
\hline \multirow[b]{2}{*}{ Treatments (ppm.) } & \multicolumn{3}{|c|}{ Wt. grains/spike (g) } & \multicolumn{3}{|c|}{ Wt. grains/plant (g) } & \multicolumn{3}{|c|}{ Wt. 1000 grain (g) } & \multicolumn{3}{|c|}{ Yield (Kg/fed.) } \\
\hline & Sakha 93 & $\begin{array}{l}\text { Giza } \\
168 \\
\end{array}$ & Mean & $\begin{array}{l}\text { Sakha } \\
93 \\
\end{array}$ & $\begin{array}{l}\text { Giza } \\
168 \\
\end{array}$ & Mean & $\begin{array}{l}\text { Sakha } \\
93 \\
\end{array}$ & $\begin{array}{l}\text { Giza } \\
168 \\
\end{array}$ & Mean & Sakha 93 & $\begin{array}{l}\text { Giza } \\
168 \\
\end{array}$ & Mean \\
\hline Control & 1.41 & 1.88 & 1.64 & 5.85 & 8.18 & 7.015 & 32.41 & 38.90 & 35.65 & 1693.4 & 1763.6 & 1728.5 \\
\hline Glutathione 50 & 1.62 & 2.14 & 1.88 & 10.81 & 15.76 & 13.28 & 35.48 & 38.87 & 37.17 & 1877.0 & 2309.0 & 2093.0 \\
\hline Glutathione 100 & 1.78 & 2.49 & 2.13 & 11.12 & 14.99 & 13.05 & 38.3 & 48.33 & 43.31 & 2541.7 & 2485.0 & 2513.3 \\
\hline Ascorbic acid 50 & 1.75 & 2.04 & 1.89 & 10.54 & 12.46 & 11.5 & 34.28 & 43.05 & 38.66 & 2264.0 & 2431.5 & 2347.7 \\
\hline Ascorbic acid 100 & 1.48 & 2.02 & 1.75 & 8.68 & 11.24 & 9.96 & 33.16 & 40.61 & 36.88 & 2205.3 & 1967.5 & 2086.4 \\
\hline Mean & \multicolumn{3}{|c|}{ Cultivars $=0.06$} & \multicolumn{3}{|c|}{ Cultivars $=0.33$} & \multicolumn{3}{|c|}{ Cultivars $=0.43$} & \multicolumn{3}{|c|}{ Cultivars $=61.5$} \\
\hline \multirow[t]{2}{*}{ LSD at $5 \%$} & \multicolumn{3}{|c|}{ Treatments $=0.11$} & \multicolumn{3}{|c|}{ Treatments $=0.70$} & \multicolumn{3}{|c|}{ Treatments $=1.57$} & \multicolumn{3}{|c|}{ Treatments $=111.3$} \\
\hline & \multicolumn{3}{|c|}{ Cultivar X treatment $=0.15$} & \multicolumn{3}{|c|}{ Cultivar X treatment $=0.9$} & \multicolumn{3}{|c|}{ Cultivar X treatment $=2.22$} & \multicolumn{3}{|c|}{ Cultivar X treatment $=157.3$} \\
\hline
\end{tabular}

Table 6. Effect of the two antioxidants glutathione and ascorbic acid on chlorophyll a, chlorophyll b and carotenoids in the leaves of the two wheat cultivars Sakha 93 and Giza 168 at vegetative and heading stages. (Average of the two seasons).

\begin{tabular}{|c|c|c|c|c|c|c|}
\hline \multirow{3}{*}{ Treatments (ppm) } & \multicolumn{6}{|c|}{ Chlorophyll a (mg/g FW.) } \\
\hline & \multicolumn{3}{|c|}{ Vegetative stage } & \multicolumn{3}{|c|}{ Heading stage } \\
\hline & Sakha 93 & Giza 168 & Mean & Sakha 93 & Giza 168 & Mean \\
\hline Control & 0.63 & 0.67 & 0.65 & 0.69 & 0.63 & 0.66 \\
\hline Glutathione 50 & 0.71 & 0.75 & 0.73 & 0.78 & 0.79 & 0.78 \\
\hline Glutathione 100 & 0.93 & 0.84 & 0.88 & 1.04 & 0.80 & 0.92 \\
\hline Ascorbic acid 50 & 0.76 & 0.83 & 0.80 & 0.83 & 0.78 & 0.80 \\
\hline Ascorbic acid 100 & 0.67 & 0.75 & 0.71 & 0.75 & 0.85 & 0.80 \\
\hline Mean & \multicolumn{3}{|c|}{ Cultivars $=0.014$} & \multicolumn{3}{|c|}{ Cultivars $=0.026$} \\
\hline \multirow[t]{3}{*}{ LSD at $5 \%$} & \multicolumn{3}{|c|}{ Treatments $=0.037$} & \multicolumn{3}{|c|}{ Treatments $=0.043$} \\
\hline & \multicolumn{3}{|c|}{ Cultivar X treatment $=0.053$} & \multicolumn{3}{|c|}{ Cultivar X treatment $=0.062$} \\
\hline & \multicolumn{6}{|c|}{ Chlorophyll b (mg/g FW.) } \\
\hline \multirow[t]{2}{*}{ Treatments (ppm) } & \multicolumn{3}{|c|}{ Vegetative stage } & \multicolumn{3}{|c|}{ Heading stage } \\
\hline & Sakha 93 & G168 & Mean & Sakha 93 & G168 & Mean \\
\hline Control & 0.37 & 0.34 & 0.36 & 0.37 & 0.35 & 0.36 \\
\hline Glutathione 50 & 0.46 & 0.55 & 0.51 & 0.47 & 0.56 & 0.52 \\
\hline Ascorbic acid 50 & 0.57 & 0.52 & 0.55 & 0.52 & 0.53 & 0.52 \\
\hline Ascorbic acid 100 & 0.35 & 0.47 & 0.41 & 0.38 & 0.48 & 0.43 \\
\hline \multirow[t]{2}{*}{ Mean } & 0.49 & 0.51 & & 0.49 & 0.52 & \\
\hline & \multicolumn{3}{|c|}{ Cultivars $=0.007$} & \multicolumn{3}{|c|}{ Cultivars $=0.006$} \\
\hline \multirow[t]{3}{*}{ LSD at $5 \%$} & \multicolumn{3}{|c|}{ Treatments $=0.033$} & \multicolumn{3}{|c|}{ Treatments $=0.017$} \\
\hline & \multicolumn{3}{|c|}{ Cultivar X treatment $=0.047$} & \multicolumn{3}{|c|}{ Cultivar X treatment $=0.023$} \\
\hline & \multicolumn{6}{|c|}{ Carotenoids (mg/g FW.) } \\
\hline \multirow[t]{2}{*}{ Treatments (ppm) } & \multicolumn{3}{|c|}{ Vegetative stage } & \multicolumn{3}{|c|}{ Heading stage } \\
\hline & Sakha 93 & Giza 168 & Mean & Sakha 93 & Giza 168 & Mean \\
\hline Control & 0.28 & 0.27 & 0.27 & 0.28 & 0.26 & 0.27 \\
\hline Glutathione 50 & 0.36 & 0.48 & 0.42 & 0.37 & 0.49 & 0.43 \\
\hline Glutathione 100 & 0.45 & 0.48 & 0.47 & 0.41 & 0.49 & 0.45 \\
\hline Ascorbic acid 50 & 0.36 & 0.40 & 0.38 & 0.46 & 0.41 & 0.44 \\
\hline Ascorbic acid 100 & 0.29 & 0.37 & 0.33 & 0.30 & 0.38 & 0.34 \\
\hline Mean & 0.35 & 0.40 & & 0.36 & 0.41 & \\
\hline & Cultivars $=$ & & & Cultivars $=$ & & \\
\hline
\end{tabular}


Table 7. Effect of the two antioxidants glutathione and ascorbic acid on total free amino acids and total phenol contents in the leaves of the two wheat cultivars Sakha 93 and Giza 168 at both vegetative and heading. (Average of the two seasons).

\begin{tabular}{|c|c|c|c|c|c|c|c|c|c|c|c|c|}
\hline \multirow{3}{*}{ Treatments (ppm.) } & \multicolumn{6}{|c|}{ Vegetative stage } & \multicolumn{6}{|c|}{ Heading stage } \\
\hline & \multicolumn{3}{|c|}{ Free amino acid (mg/g DW) } & \multicolumn{3}{|c|}{ Total phenol (mg/g DW) } & \multicolumn{3}{|c|}{ Free amino acid (mg/g DW) } & \multicolumn{3}{|c|}{ Total phenol (mg/g DW) } \\
\hline & Sakha 93 & Giza 168 & Mean & Sakha 93 & Giza 168 & Mean & Sakha 93 & Giza 168 & Mean & Sakha 93 & Giza 168 & Mean \\
\hline Control & 15.82 & 20.02 & 17.92 & 14.16 & 14.47 & 14.31 & 10.19 & 12.38 & 11.28 & 12.51 & 11.17 & 11.84 \\
\hline Glutathione 50 & 18.86 & 20.83 & 19.85 & 14.90 & 15.37 & 15.13 & 13.12 & 14.00 & 13.56 & 11.20 & 12.61 & 11.90 \\
\hline Glutathione 100 & 21.18 & 25.99 & 23.59 & 12.61 & 15.4 & 14.00 & 16.86 & 17.43 & 17.14 & 11.56 & 11.29 & 11.42 \\
\hline Ascorbic acid 50 & 19.80 & 24.31 & 22.06 & 13.92 & 16.46 & 15.19 & 13.52 & 15.40 & 14.46 & 13.18 & 12.52 & 12.85 \\
\hline Ascorbic acid 100 & 18.82 & 23.67 & 21.25 & 14.16 & 15.09 & 14.62 & 11.29 & 13.21 & 12.25 & 9.93 & 12.1 & 11.01 \\
\hline Mean & \multicolumn{3}{|c|}{ Cultivars $=0.19$} & \multicolumn{3}{|c|}{ Cultivars $=0.25$} & \multicolumn{3}{|c|}{ Cultivars $=0.15$} & \multicolumn{3}{|c|}{ Cultivars $=0.15$} \\
\hline \multirow[t]{2}{*}{ LSD at $5 \%$} & \multicolumn{3}{|c|}{ Treatments $=1.12$} & \multicolumn{3}{|c|}{ Treatments $=0.91$} & \multicolumn{3}{|c|}{ Treatments $=0.92$} & \multicolumn{3}{|c|}{ Treatments $=0.67$} \\
\hline & \multicolumn{3}{|c|}{ Cultivar $X$ treatment $=1.58$} & \multicolumn{3}{|c|}{ Cultivar $\mathrm{X}$ treatment $=1.28$} & \multicolumn{3}{|c|}{ Cultivar X treatment $=1.29$} & \multicolumn{3}{|c|}{ Cultivar $X$ treatment $=0.94$} \\
\hline
\end{tabular}

Table 8. Effect of the two antioxidants glutathione and ascorbic acid on the percentage of total carbohydrate and protein contents in the leaves of the two wheat cultivars Sakha 93 and Giza 168 at both vegetative and heading. (Average of the two seasons).

\begin{tabular}{|c|c|c|c|c|c|c|c|c|c|c|c|c|}
\hline \multirow{3}{*}{ Treatments (ppm.) } & \multicolumn{6}{|c|}{ Vegetative stage } & \multicolumn{6}{|c|}{ Heading stage } \\
\hline & \multicolumn{3}{|c|}{ Total carbohydrates \% } & \multicolumn{3}{|c|}{ Protein \% } & \multicolumn{3}{|c|}{ Total carbohydrates \% } & \multicolumn{3}{|l|}{ Protein \% } \\
\hline & Sakha 93 & Giza 168 & Mean & Sakha 93 & Giza 168 & Mean & Sakha 93 & Giza168 & Mean & Sakha 93 & Giza168 & Mean \\
\hline Control & 40.6 & 38.6 & 39.60 & 11.9 & 12.4 & 12.15 & 32.0 & 43.3 & 37.65 & 11.5 & 11.7 & 11.60 \\
\hline Glutathione & 44.9 & 51.0 & 47.95 & 12.9 & 13.8 & 13.35 & 53.8 & 58.6 & 56.20 & 12.6 & 12.8 & 12.70 \\
\hline Glutathione & 53.6 & 76.7 & 65.15 & 13.6 & 15.4 & 14.50 & 58.1 & 71.3 & 64.70 & 13.2 & 13.7 & 13.45 \\
\hline Ascorbic acid 50 & 48.9 & 51.3 & 50.10 & 14.5 & 14.8 & 14.65 & 51.4 & 62.3 & 56.85 & 14.4 & 12.1 & 13.25 \\
\hline Ascorbic acid & 42.5 & 40.4 & 41.45 & 13.8 & 12.4 & 013.1 & 37.6 & 52.6 & 045.1 & 14.1 & 13.6 & 13.85 \\
\hline Mean & \multicolumn{3}{|c|}{ Cultivars $=0.40$} & \multicolumn{3}{|c|}{ Cultivars $=\mathrm{NS}$} & \multicolumn{3}{|c|}{ Cultivars $=0.19$} & \multicolumn{3}{|c|}{ Cultivars $=0.29$} \\
\hline \multirow[t]{2}{*}{ LSD at $5 \%$} & \multicolumn{3}{|c|}{ Treatments $=0.74$} & \multicolumn{3}{|c|}{ Treatments $=0.62$} & \multicolumn{3}{|c|}{ Treatments $=0.96$} & \multicolumn{3}{|c|}{ Treatments $=0.61$} \\
\hline & \multicolumn{3}{|c|}{ Cultivar X treatment $=1.05$} & \multicolumn{3}{|c|}{ Cultivar X treatment $=0.87$} & \multicolumn{3}{|c|}{ Cultivar X treatment $=1.36$} & \multicolumn{3}{|c|}{ Cultivar X treatment $=0.86$} \\
\hline
\end{tabular}

The increase in the photosynthetic pigments (Table 6) in the two wheat cultivars is associated with the increase in the percentage of carbohydrate (Table 8). The results in Table 8 indicate that Glut. at $100 \mathrm{ppm}$ caused significant increase in carbohydrate content as compared to the corresponding control. In addition, total protein content were quantitatively increased at $100 \mathrm{ppm}$ Glut. in the leaves of the two cultivars (Table 8).

\section{Discussion}

Glutathione is crucial for biotic and abiotic stress management. It is a pivotal component of glutathioneascorbate cycle, a system that reduces poisonous hydrogen peroxide [11]. Glutathione is required for efficient defense against plant pathogens [22]. It is used as electron donor in APS reductase (adenosine 5-phosphosulfate reductase), an enzyme of the sulfur assimilation pathway. Glutathione also is used as substrate in other enzymes as glutaredoxin, these small oxidoreductases are involved in flower development, salicylic acid and plant defense signaling [23].

Ascorbate is a major metabolite in plants. It is an antioxidant and, in association with other components of the antioxidant system, protects plants against oxidative damage resulting from aerobic metabolism, photosynthesis and a range of pollutants [24].

In the present results, when the two wheat cultivars Sakha 93 and Giza168 foliarly treated with Glut. or ASA performed similar trend in responses of most criteria of growth. The two cultivars were highly significantly increased in growth i.e plant height as well as fresh and dry weight of shoot (Table 2). The two cultivars showed great responses to higher concentration $100 \mathrm{ppm}$ of Glut. whereas showing high growing rate with the lower concentration $50 \mathrm{ppm}$ of ASA. Previous studies have demonstrated that ASA. increased wheat growth $[4,5,6]$. It have been reported that Glut. also increased plant growth [25 and 26]. The increase in plant growth by Glut. or ASA. may be attributed to the enhancement of cell division [27]. Noctor et al. [28] referred that plants cannot survive without glutathione that has functions in plant development which cannot be performed by other thiols or antioxidants.

Enhancement of the two wheat cultivars growth by Glut. or ASA. was associated with parallel increase in yield expressed as increased number of spikes per plant, number of spiklets per spike and grain yield per spike, weight of 1000 grains (Tables3\&4). These reflected on significant increases in the grain yield (yield/fed.). EL-Hosary et al. [29] reported that treatment of wheat cultivar (Sakha 93) with ascorbic acid at $100 \mathrm{mg} / 1$ resulted in significant increase in grain yield. In addition, Mohamed [30] obtained significant growth, grain yield and its components of wheat cv.Masr-1 in response to foliar application of ascorbic acid.

The importance of glutathione in its involvement in the formation of phytochelatins and together with its oxidized form (GSSG) make up the redox couple (GSH/GSSG), which plays an essential role in maintenance of the cellular homeostasis and signaling system in plants [31]. Consequently, importance of glutathione may be attributed to that it is roles in biosynthetic pathways, detoxification, antioxidant 
biochemistry and redox homeostasis [28].

Increased metabolic activity of wheat plants could be attributed to the resulted corresponding increase in photosynthetic pigments (Table 6) consequently the content of carbohydrate [32] in addition to increase of protein synthesis (Table 8). The increase in the biosynthesis of total free amino acids (Table 7) may be the cause of increasing protein biosynthesis [33]. Based on the above conclusion of several workers, as well as the results in Tables 6,7 and 8 the increases in metabolic activities may be attributed to Glutathione role in biosynthetic pathways, detoxification, antioxidant biochemistry and redox homeostasis [28 and 31]. In addition, Al-Muwayh [34] reported increase in protein synthesis of Phaseouls vulgaris L. by various concentrations of ascorbic acid.

\section{References}

[1] Bolkhina, O., Virolainen, E., Fegerstedt K.V. 2003. Antioxidant, oxidative damage and oxygen deprivations stress. A Review Ann. Bot. 91,179-194.

[2] Kumar V., Lemos M., Sharma, M., Shriram, V. 2013. Antioxidant and DNA damage protecting activities of Eulophia nuda Lindl. Free Radicals and Antioxidants. 3(2), 55-60.

[3] El-Kobisy, D.S., Kady, K.A., Medani, R.A., Agamy, R.A. 2005 Response of pea plant (Pisum sativum L.) to treatment with ascorbic acid. Egypt. J. Appl. Sci. 20, 36-50.

[4] Negm, A.Y. 1998. Foliar application of ascorbic acid and copper to wheat plants grown on sandy soils. Egypt. J. Appl. Sci. 13(5), 312-319.

[5] Amin, A.A., Rashad, El-Sh.M. Gharib, F.A.E. 2008. Changes in morphological, physiological and reproductive characters of wheat plants as affected by foliar application with salicylic acid and ascorbic acid. Austr. J. Basic and Appli. Sci. 2(2), 252-261.

[6] Bakry, A.B., Abdelraouf, R.E., Ahmed, M.A., El Karamany, M.F. 2012. Effect of drought stress and ascorbic acid foliar application on productivity and irrigation water use efficiency of wheat under newly reclaimed sandy soil. J. Appl. Sci. Res. $8(8), 4552-4558$.

[7] Gamal El-Din, K.M, Reda, F. 2003. Effect of presowing heat hardening and ascorbic acid treatments on growth, yield and some biochemical constituents of wheat (Triticum aectivum L.). Egypt. J. Appl. Sci. 18(12), 91-105.

[8] Amin, B., Mahleghah, G., Mahmood, H.M.R., Hossein, M. 2009. Evaluation of interaction effect of drought stress with ascorbate and salicylic acid on some physiological and biochemical parameters in Okra (Hibiscus esculentus L.). Res. J. Biol. Sci. 4: 380-387.

[9] Pompella, A., Visvikis, A., Paolicchi, A., Tata, V., Casini, A.F. 2003. The changing faces of glutathione, a cellular protagonist. Bioch. Pharm. 66 (8), 1499-503.

[10] Asada, K., Takahashi, M. 1987. Production and scavenging of active oxygen in photosynthesis. In Photoinhibition. Edited by Kyle, D.J., Osmond, C.B. and Arntzen, C.J. Elsevier, Amsterdam, pp. 227-287. Noctor, G. Foyer, C.H. 1998. Ascorbate and glutathione: keeping active oxygen under control. Annu. Rev. Plant Physiol. Plant Mol. Biol. 49, 249-279.
[11] Noctor, G. Foyer, C.H. 1998. Ascorbate and glutathione: keeping active oxygen under control. Annu. Rev. Plant Physiol. Plant Mol. Biol. 49, 249-279.

[12] Henmi, K., Tsuboi, S., Demura, T., Fukuda, H., Iwabuchi, M., Ogawa, K. 2001. A possible role of glutathione and glutathione disulfide in tracheary element differentiation in the cultured mesophyll cells of Zinnia elegans. Plant Cell Physiol. 42, 673-676.

[13] Ogawa, K., Hatano-Iwasaki, A., Yanagida, M. Henmi, K. 2002. The redox-dependent regulation of bolting and flowering in rosette-type plants. Flowering Newslett. 34, 45-51.

[14] Ogawa, K., Hatano-Iwasaki, A., Yanagida, M. Iwabuchi, M. 2004. Level of glutathione is regulated by ATP-dependent ligation of glutamate and cysteine through photosynthesis in Arabidopsis thaliana: mechanism of strong interaction of light intensity with flowering. Plant Cell Physiol. 45, 1-8.

[15] Wettstein, D. 1957. Chlorophyll lethal und der submikroskopiche Formwechsel der plastiden. Cell. Res. 12 (3), 427-433.

[16] A. O.A.C. 2000. Official Methods of Analysis of Association of official chemists. Chlorophyll in plants, spectrophotometric method. $17^{\text {th }}$ ed. Helrich K. (Ed) Inc: Arlingten, Virginia, USA., pp 28.

[17] A.O.A.C. 1990. Official Methods of Analysis of Association Agriculture Chemists. $15^{\text {ed }}$. Washington, USA.

[18] Plummer, D. (1978). An introduction to practical biochemistry. $2^{\text {nd }}$ Ed. McGraw-Hall Mook Company Limited, London, UK., 144.

[19] Snell, F.D., Snell, C.T. 1953. Colorimetric Methods. Volume III. Organic, D. Van Nostrand Company, Inc. Toronto, New York, London, $606 \mathrm{pp}$.

[20] Dubois, M., Guilles, K.A, Hamilton, J.K., Rebers, P.A., Smith, F. 1956. Colorimetric method for determination of sugars and related substances. Anal. Chem. 28, 350-356.

[21] Snedecor, G.W., Cochran, W.G. 1980. Statistical Methods. $7^{\text {th }}$ ed. The Iowa State Univ. Press, Ames, Iowa, I A., 507 pp.

[22] Parisy, V., Lucas, O., Antony, B., Jane, G., Felix, M. 2006. Identification of PAD2 as a $\gamma$-glutamylcysteine synthetase highlights the importance of glutathione in disease resistance of Arabidopsis. The Plant J. 49 (1), 159-72.

[23] Rouhier, N., Lemaire, S.D., Jacquot, J.P. 2008. The role of glutathione in photosynthetic organisms: Emerging functions for glutaredoxins and glutathionylation. Annu. Rev. of Plant Biol. 59, 143-66.

[24] Smirnoff, N. 1996. The function and metabolism of ascorbic acid in plants. Ann. Bot.78, 661- 669 .

[25] Franceschi, V.R., Tarlyn, N.M. 2002. L-ascorbic acid is accumulated in source leaf phloem and transported to sink tissues in plants. Plant Physiol. 130 (2), 649-656.

[26] Talaat, I.M., Aziz, E.E. 2005. Stimulatory effects of glutathione, nicotinic acid and ascorbic acid on matricaria. Egypt. J. applied Sci. 20, 218-231.

[27] Sanchez-Fernández, R., Fricker, M., Corben, L.B., White, N.S., Sheard, N., Leaver, C., Montagu, M.V., Inzé, D., May, M.J. 1997. Cell proliferation and hair tip growth in the Arabidopsis root are under mechanistically different forms of redox control. Proc. Natl Acad. Sci. USA. 94, 2745-2750. 
[28] Noctor, G., Mhamdi, A., Chaouch, S., Han, Y., Neukermans, J., Marquez-Garcia, B., Queval, G., Foyer, C.H 2012. Glutathione in plants: an integrated overview. Plant Cell Environ. 35(2):454-484

[29] EL-Hosary, A.A., Hammam, G.Y., EL-Morsi, A., Hassan, E.A., EL-Awadi, M.E., Abdel-Baky Y.R. 2013. Effect of some Bio-regulators on growth and yield of some wheat varieties under newly cultivated land conditions. New York Sci.J. 6(6), $1-11$.

[30] Mohamed, N.E.M. 2013. Behaviour of wheat Cv. Masr-1 plants to foliar application of some vitamins. Nat. and Sci. 11(6), 1-5.

[31] Srivalli, S., Khanna-Chopra, R. 2008. Delayed wheat flag leaf senescence due to the removal of spikelets is associated with increased activities of leaf antioxidant enzymes, reduced glutathione/oxidized glutathione ratio and oxidative damage to mitochondrial proteins. Plant Physiol. Biochem. 47, 663-670.

[32] Coruzzi, G., Last, R., 2000. Amino acids. In: Biochemistry and molecular biology of plants. B. Buchanan, Gruissem, R. Jones (eds.). American Society of Plant Physiologists (pubs.). Maryland, USA. pp: $358-410$

[33] El-Rokiek, K.G, El-Awady, M.E., Abd El-Wahed, M.S.A. 2012. Physiological responses of wheat plants and accompanied weeds to derby herbicide and $\beta$-sitosterol bioregulator. J. Appl. Sci. Res., 8(4), 1918-1926.

[34] Al-Muwayh, M.A. 2012. Effect of ascorbic acid on lipid and protein contents of Phaseouls vulgaris L grown at gaseous polluted areas in Riyadh city, Saudi Arabia. New Horizons in Sci. \& Technol. (NHS\&T). 1(3), 65-70 